\title{
Special topic on research frontier in polymer science in China
}

\author{
Anjun Qin $^{1 *} \&$ Ben Zhong Tang ${ }^{1,2 *}$ \\ ${ }^{1}$ State Key Laboratory of Luminescent Materials and Devices, South China University of Technology, Guangzhou 510640, China \\ ${ }^{2}$ Department of Chemistry, Hong Kong Branch of National Engineering Research Center for Tissue Restoration and Reconstruction, \\ The Hong Kong University of Science \& Technology, Clear Water Bay, Kowloon, Hong Kong, China
}

Received November 4, 2016; accepted November 6, 2016; published online November 14, 2016

Citation: $\quad$ Qin A, Tang BZ. Special topic on research frontier in polymer science in China. Sci China Chem, 2016, 59: 1529-1530, doi: 10.1007/s11426-016-0447-4

Polymer science is a multiple discipline that studies synthesis, structure, function and application of macromolecules. It encompasses subdisciplines of polymer chemistry, physics and engineering. Despite the fact that polymeric materials had been known and used for quite long time, polymer chemistry was not established until Hermann Staudinger coined the concept of "Makromoleküle" in the 1920s. From then on, polymer chemistry developed quickly, which subsequently gave birth to polymer physics and engineering. As a result, polymer science was finally formed. Nowadays, the achievement in polymer science has greatly promoted the development of our world and been part of our civilization.

The research on polymer science in China started in the early 1950s. With the outstanding contributions from the domestic polymer scientists, our country is playing more and more important role in the field of polymer science and some breakthroughs in the areas of synthetic polymer chemistry, optoelectronic polymers, biomacromolecules, etc., have been achieved. To highlight the fruitful outcome in the polymer science in our country, we edit this special topic of Frontiers in Polymer Science for Science China Chemistry, with the aim to stimulate continuous innovation in the field.

The contents of this special topic cover the current hot research topics including polymer synthesis, supramolecular polymers, optoelectronic polymers and biomacromolecules

*Corresponding authors (email: msqinaj@scut.edu.cn; tangbenz@ust.hk) in the field of polymer research.

In the area of synthetic polymer chemistry, Tao et al. briefly accounted the applications of the Biginelli reaction in functional polymer preparation. Qin, Tang et al. reported the utilization of their established metal-free click polymerization of activated alkyenes and azides in the preparation of hyperbranched polytriazoles with self-healing properties.

In the supramolecular polymer area, Liu et al. summarized the progress on the engineering and application in the biomedical areas of supramolecular protein architectures. Sun et al. fabricated a smart film by depositing hydrophobic $\mathrm{SiO}_{2}$ nanoparticles on poly(acrylic acid)/poly-(allylamine hydrochloride) films, followed by partially exfoliating the films from the underlying substrates. The film could reversibly change its wettability between transparent hydrophobicity to translucent superhydrophobicity through the humidity-induced wrinking/de-wrinkling process. Chen et al. reported the supramolecular modification of the conjugated polyfluororene nanoparticles bearing cyclodextrin on their surfaces via host-guest interaction between cyclodextrin and functional-group containing adamantine. The resultant glycopolymer-modified conjugated polymer nanoparticles showed excellent binding ability to lectins and selective imaging behavior to Hep G2 cells.

In the optoelectronic polymer aspect, Hou et al. successfully realized $11.3 \%$ efficiency in fullerene-free polymer solar cells through systematical device optimization. Duan, Huang et al. developed a series of new acceptor-pended conjugated polymers, which feature a novel carbazolederived building unit for polymer solar cells. Li et al. de- 
scribed the preparation of two soluble dendronized hyperbranched polymers by " $\mathrm{A}_{3}+\mathrm{B}_{2}$ " type Sonogashira polycoupling. The nonlinear optical coefficients were as high as 220 $\mathrm{pm} / \mathrm{V}$ and high orientation stability of these polymers were realized. Ding, Wang et al. developed a series of self-host yellow Ir dendrimers and a peak luminous efficiency of 20.2 cd/A was obtained in the second generation polymer. This value could be enhanced to $32.1 \mathrm{~cd} / \mathrm{A}$ when this polymer was dispersed into a host matrix.

In the biomacromolecule area, Ren et al. reviewed the existing methods for preparing collagen based corneal regeneration materials. Deng, Zhu et al. presented dual-modal surface enhanced Raman spectrum (SERS)-fluorescence polymer/metal hybrid complexes for tracing drug release process in tumor cells. Tang, Ji et al. discussed pH-triggered theranostic hyaluronic acid prodrug micelles containing biomimetic phosphorylcholinine, anticancer drug doxorubicin and tetraphenylethene moieties. Such theranostic drug delivery system with aggregation-induced emission features provides a new platform for targeted and image-guided cancer therapy.

The above works have shed light on the bright future of polymer science in China. We are confident that great progress will be made with continue efforts devoted by our polymer scientists, and more and more innovative and original results will be presented. These achievements will in turn make great contributions to the science \& technology development as well as social advancement in China.

We would like to thank all the contributors and reviewers for their time and efforts. We appreciate Dr. Jianfen Zheng from the editorial office of Science China Chemistry for her professional support.

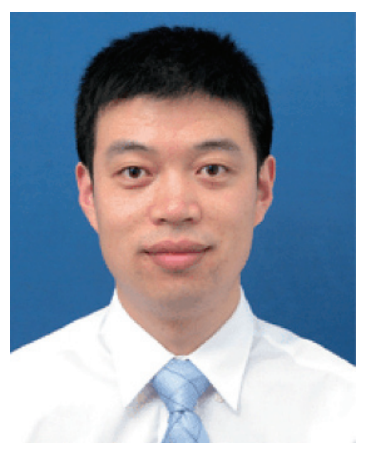

Prof. Anjun Qin received his BSc and PhD degrees from Shanxi University and Institute of Chemistry of the Chinese Academy of Sciences in 1999 and 2004, respectively. He conducted his postdoctoral research at the Hong Kong University of Science \& Technology (HKUST) and Zhejiang University (ZJU) under the supervision of Prof. Ben Zhong Tang during 2005-2008. He joined ZJU as an Associate Professor at the end of 2008 and moved to South China University of Technology with promotion to Full Professor in 2013. His current research interests include the development of new polymerizations based on triple-bond building blocks, and the construction of organic/polymeric functional materials.

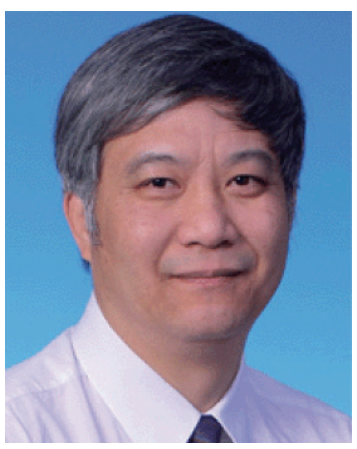

Prof. Ben Zhong Tang received his PhD degree from Kyoto University in 1988. He conducted his postdoctoral work at University of Toronto in 1989-1994. He joined HKUST in 1994 and was promoted to Chair Professor in 2008 and Stephen K. C. Cheong Professor of Science in 2013. He was elected to the Academician of Chinese Academy of Sciences in 2009. His research interests include the exploration of new polymerization based on triple-bond building blocks and investigation of aggregation-induced emission. 